\title{
Fifteen-minute consultation: Insulin pumps for type 1 diabetes in children and young people
}

\author{
Philippa Prentice, ${ }^{1}$ Daniela Elleri ${ }^{2}$
}

\begin{abstract}
'Department of Paediatrics, North Middlesex University Hospital NHS Trust, London, UK ${ }^{2}$ Department of Paediatric Endocrinology, Royal Hospital for Sick Children, Edinburgh, UK
\end{abstract}

\section{Correspondence to}

Dr Philippa Prentice, Department of Paediatrics, North Middlesex Hospital, Sterling Way, London N18 1QX, UK; philippa. prentice1@nhs.net

Received 24 May 2017 Revised 20 September 2017 Accepted 21 September 2017 Published Online First 3 January 2018
Check for updates

To cite: Prentice P, Elleri D. Arch Dis Child Educ Pract Ed 2018;103:131-136.

\begin{abstract}
There is increasing worldwide use of continuous subcutaneous insulin infusions in paediatric type 1 diabetes (T1D), reflecting recent research outcomes and guidance, as well as families' wishes. Children/young people may present acutely with medical or surgical problems, in addition to issues related to T1D. This review provides general paediatricians with an introduction to pump therapy, highlighting common problems, management issues and when to seek specialist advice.
\end{abstract}

\section{INTRODUCTION}

Type 1 diabetes (T1D) is one of the most common chronic diseases in childhood and adolescence. It still causes significant long-term morbidity and mortality, and its incidence is increasing worldwide, particularly in younger children. ${ }^{1}$ The Diabetes Control and Complication Trial and follow-up data demonstrated that intensive insulin therapy, using multiple daily injections (MDI) or pump therapy, improved glucose control and reduced microvascular complications in adolescents and young adults. ${ }^{23}$

Continuous subcutaneous insulin infusion (CSII), using a portable infusion pump, was first shown to be effective in the $1970 \mathrm{~s}^{4}$ and is now widely used in paediatric practice. A continuous but varying amount of rapid-acting insulin is delivered subcutaneously, mirroring physiological insulin release more closely than MDI. Therefore, its aims are to further reduce hyperglycaemia and subsequent complications, decrease episodes and associated fear of hypoglycaemia, and improve quality of life for children and their families.

Despite worldwide variation, international guidelines and registry data support CSII use, ${ }^{5}$ and many families are keen to have a pump. A 2012 UK audit identified CSII use in 19\% of children and young people, ${ }^{6}$ and this is likely to have increased. In the USA over $60 \%$ of children aged 2-12 years old with T1D use CSII. ${ }^{7}$ Therefore, increasing numbers of children and young people using CSII will present to hospital with a range of problems, related or unrelated to T1D.

CSII requires a multidisciplinary team approach and teaching. Many units have dedicated out-of-hours advice and this should be sought. However, it is important for all paediatricians to be aware of the common scenarios faced with CSII, know how to initiate early management and be aware of when to seek specialist advice. This article aims to provide an overview of how CSII works, discuss common problems and their management, and signpost to some available resources (box 1). Details of initiating pump therapy, starting insulin requirements and more complex management are discussed elsewhere. ${ }^{89}$

\section{OVERVIEW OF CSII AND WHAT CAN GO WRONG}

What is an insulin pump and how does it work?

CSII is made up of an insulin reservoir (typically storing 176-300 units of insulin), an infusion set with a small cannula-inserted subcutaneously and made of plastic or metal, a battery-operated motor and an electromechanical pump with a computerised control. Multiple sites (abdomen, outer thigh, hips, buttocks and top of the arms) can be used to insert the infusion set cannula and sites should be rotated. Continuous rapid-acting insulin is delivered subcutaneously-the 'basal' insulin-which can be set to any amount (as little as 0.025 units per hour for most pumps; 0.01 units per hour with the Roche Insight pump), and so ideal for any-sized child, including infants. Basal insulin is varied throughout the day and night, reflecting physiological 


\section{Box 1 Resources}

- Insulin pumps and continuous glucose monitoring made easy ${ }^{8}$

- Carbohydrate counting: www.carbsandcals.com

- Exercise with type 1 diabetes: www.runsweet.com

- A few useful apps:

Carbs \& Cals

Cook \& Count

Fitness Pal

variation in fasting insulin requirements. Additionally, 'bolus' insulin is given before meals, and as 'correction doses' in hyperglycaemia, via the computerised handset device. Most families use bolus calculators incorporated into the CSII, entering carbohydrate intake, along with their current blood glucose (BG) reading, to calculate insulin doses. The handset display allows recall of information, such as basal insulin rates throughout the day and total daily doses of insulin. Further terminology is explained in box 2 .

\section{Is using CSII better than other insulin regimens?}

Few randomised controlled trials or long-term data exist in the paediatric population. Therefore, most guidance results from expert opinion and observational studies. ${ }^{10}$ Although still disputed,${ }^{11}$ the majority of systematic reviews, meta-analyses and guideline recommendations suggest that CSII results in lower risk of hypoglycaemia, lower insulin requirements, and better glycaemic control in children and young people, although improved glucose control also depends on glycated haemoglobin (HbA1c) before commencing CSII. ${ }^{12}{ }^{13}$ Recent data from the SWEET (Better control in Pediatric and Adolescent diabeteS: Working to crEate CEnTers of Reference) project, including 16570 children across 46 international centres, demonstrated lower $\mathrm{HbA} 1 \mathrm{c}$ in children of all age groups using CSII, compared with MDI. ${ }^{14}$

\section{So when should insulin pumps be used?}

The National Institute for Health and Care Excellence (NICE) guidance (technology appraisal guidance 151, 2008) recommends CSII therapy as a treatment option for $\mathrm{T}_{1} \mathrm{D}^{15}$ in:

- children of 12 years or older when HbA1c levels remain high $(69 \mathrm{mmol} / \mathrm{mol}$ or above $(8.5 \%))$ using MDI, despite a high level of care

- children of 12 years or older with disabling hypoglycaemia-repeated or unpredictable episodes of hypoglycaemia causing persistent anxiety and reduced quality of life, when trying to achieve HbA1c targets with MDI

- children under 12 years when MDI is deemed 'impractical' or 'inappropriate'.

International recommendations do not include these age ranges. The global International Diabetes Federation/International Society for Pediatric and Adolescent Diabetes guideline (2011) suggested that CSII should be available and considered for all children and young

\section{Box 2 Terminology}

- $\mathrm{HbA1C}$ - glycated haemoglobin, giving a measure of average BG level over the previous 8-12 weeks; HbA1c target (NICE): $48 \mathrm{mmol} / \mathrm{mol}=6.5 \%$

\section{Pumps and sensors}

- CSII-continuous subcutaneous insulin infusion or insulin pump

- Patch pump-tubing-free insulin pump

- Continuous glucose monitoring sensor (CGMS) subcutaneously inserted device measuring interstitial glucose levels continuously

- Sensor-augmented pump-combines CSII with CGMS, allowing the user to know real-time frequent BG levels, and can also allow automated pump suspension in hypoglycaemia

- Artificial pancreas system - closed-loop system where BG levels are continuously monitored and the insulin delivered is automatically adjusted, using the systems algorithm; this is currently being used in research studies, either with insulin or using insulin and glucagon together

Insulin

- Bolus insulin-insulin dose given at a specific time point (before meals or to correct a high BG level); should not be given more frequently than 2 hourly

- Basal insulin - continuous 'background' insulin (suppressing hepatic glucose production)

- Insulin carbohydrate ratios-amount of carbohydrate (in grams) 1 unit of insulin should be given for, for example, 1:10 ratio at breakfast- -1 unit of insulin should be given for every $10 \mathrm{~g}$ of carbohydrate eaten at breakfast time

- Correction dose/insulin sensitivity factor (ISF) - predicted reduction in glucose level with 1 unit insulin, for example, 1:8-1 unit of insulin should reduce the BG level by $8 \mathrm{mmol} / \mathrm{L}$

- 'Insulin on board'—amount of insulin still active from previous recent bolus doses

BG, blood glucose; NICE, National Institute for Health and Care Excellence.

people. ${ }^{16}$ Previously, the first international consensus statement (2007) also recommended that all children with T1D were candidates, but particularly those with large BG fluctuations, microvascular complications (and/or risk factors for macrovascular complications), current treatment compromising lifestyle, recurrent severe hypoglycaemia or suboptimal HbA1c. ${ }^{10}$

It is also important to discuss the relative merits of both CSII and MDI with the child/young person and their family. Advantages of CSII include fewer injections, more flexibility and the aim of improved glycaemic control; potential disadvantages are the complexity and education needed, constant attachment to the pump and frequent BG level checking. ${ }^{9}$

\section{Choice of CSII systems and how do they differ}

There are many CSII systems available, made by different companies, including Animas (Johnson \& 
Johnson), Cellnovo, Medtronic, Omnipod, Roche, Sooil, Ypsomed. Children/young people and their families may have a choice, and decisions might be influenced by the design of the computerised handset device, tubing, cannula types, colour and whether the pump is waterproof. The majority of pumps use a subcutaneous cannula; however, an alternative is the 'patch pump', which attaches directly to the skin, and therefore needs no tubing.

Glucometer devices may be integrated into or be separate from the CSII. The insulin reservoir is either loaded with prefilled insulin cartridges or the child/ carer needs to fill the insulin cartridge manually, as per the manufacturer's instructions. There are variations in algorithms to calculate insulin boluses, set by the manufacturers, for example in the prediction models used for glucose rise after meals, whether the pump corrects to a high/low BG target or to mid-range, and how the algorithm interprets 'insulin on board' if multiple insulin boluses are given (box 2).

Some pumps can upload glucose levels (generally every $5 \mathrm{~min}$ ) from subcutaneous continuous glucose monitoring sensors. 'Sensor-augmented' pumps are provided with features that also allow automatic suspension of insulin delivery to prevent hypoglycaemia. Closed-loop artificial pancreas systems provide automated insulin delivery by a control algorithm, which uses sensor glucose readings to direct the insulin infusion via the pump. Closed-loop systems are the hope for the future, and trials have shown improved glucose control, reduction in hypoglycaemia and patient satisfaction. ${ }^{17}$

\section{What can go wrong with an insulin pump?}

Insulin delivery can be disrupted by pump malfunction or dislodgement of the cannula, causing insulin leakage. The pump mechanism can fail or the battery run down, and handsets can become damaged, for example by water (although some pumps are water-resistant) or breakage of the screen. Insulin delivery can be obstructed due to an occlusion, air bubble or kinking of the tubing. ${ }^{8} 18$

\section{THE CHILD/YOUNG PERSON PRESENTING ACUTELY}

Children/young people using CSII may present acutely with hyperglycaemia or hypoglycaemia, or with an unrelated medical or surgical condition. In all scenarios some knowledge about the pump, insulin requirements and $B G$ values is needed.

\section{KEY QUESTIONS TO ASK}

The following questions may be relevant, tailoring to specific problems:

\section{BG readings and insulin given \\ $\checkmark$ What is the current BG? \\ What has the recent trend of BG values been?}

- Have they had any hypos? What treatment was given?

- How much insulin have they given today? Has there been any insulin:carbohydrate mismatch, for example, any carbohydrate eaten without giving insulin?

- When did they last give an insulin bolus?

- Have correction boluses been given?

- What has their oral intake been like?

\section{Blood ketones}

What is the ketone level and what has the trend been? Blood ketones must always be checked if unwell (regardless of the $B G)$ and with hyperglycaemia $(B G>14 \mathrm{mmol})$.

\section{Other factors}

- Any recent travel, altering the time on the insulin pump to local time, which has not been changed back.

- Recent exercise-this is a complex area, and depending on the type and intensity of different sports can cause either hypoglycaemia or hyperglycaemia. Effects can also be immediate or much later, even affecting BG levels the subsequent day.

\section{ACUTE MANAGEMENT}

Children and their families will be given 'sick day rules' advice to follow and should contact the diabetes on-call team when unsure. It is important to remember that insulin should never be stopped even when a child is unwell, but may need adjusting. If a child presents to hospital, specialist advice should still be sought, including out-of-hours.

An overview of management is discussed below and in figure 1 and figure 2.

\section{The child in DKA}

The management of DKA must be the same as with any child, following national guidelines. ${ }^{19}$ Since CSII delivers rapid-acting insulin alone, with no long-acting insulin, children are potentially at greater risk of DKA, than those on MDI, if the CSII stops working or when they are disconnected from the pump. The risk of DKA is high after 4-6hours, but blood ketones may start rising 1-2 hours after disconnection.

CSII should be stopped when intravenous insulin is started. When subcutaneous insulin is restarted, CSII should be started at least an hour before stopping intravenous insulin. The insulin cartridge and infusion set should be changed, with a new cannula inserted.

\section{The child with hyperglycaemia ( $B G>14 \mathrm{mmol} / \mathrm{L}$ ) who is not in DKA}

A child/young person presenting with hyperglycaemia may be unwell and temporarily be needing more insulin, or have a faulty CSII system. Ketone levels must always be checked in hyperglycaemia, ideally with blood ketone strips, as recommended by NICE guidance (2015) (figure 1). ${ }^{20}$

\section{Intercurrent illness}

During acute illness, even with reduced oral intake, additional insulin is likely to be needed. Families should follow 'sick day rules', often as flow charts, showing 
what to do depending on BG and ketone levels, and national guidance should be followed. ${ }^{21}$

Extra insulin can be given as bolus doses and/or by increasing the CSII basal insulin rate. An initial correction bolus can be given via the pump, using the bolus calculator (which uses the insulin sensitivity factor (ISF)). However, with no improvement or high/rising ketone levels, a bolus of rapid-acting insulin should be given with an insulin 'pen' and the CSII infusion set changed. All children/young people using CSII will have a rapid-acting insulin 'pen' for this reason. If families are unsure of the ISF, a correction dose can be estimated from the TDD, found on the CSII monitor (box 3). With high/rising ketone levels it is appropriate to give $10 \%-20 \%$ of TDD, depending on the ketone value. $^{21}$ It is important to remember that insulin boluses can be given 2 hourly but not more frequently to avoid 'insulin stacking' (where previous insulin is still active). Additionally an increased temporary basal rate may need to be set (125\%-200\%). All increases in insulin should be discussed with the diabetes team.

For any unwell child with T1D, frequent BG and ketone monitoring (hourly-2 hourly) should continue, and fluids encouraged to avoid dehydration and increase renal excretion of ketone bodies, using sugar-free fluids in hyperglycaemia. If there is no

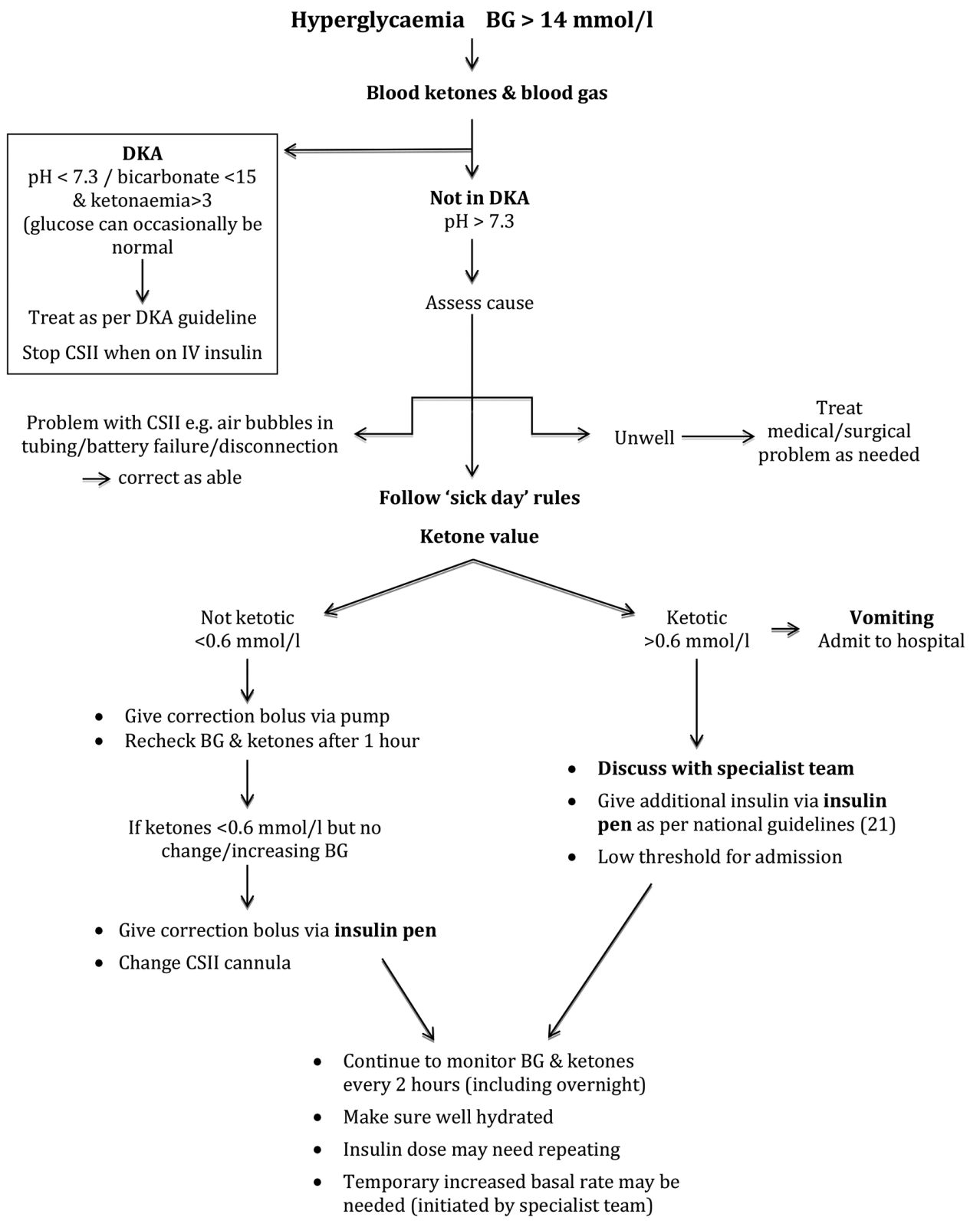

Adapted from: BSPED guideline: Management of Type 1 Diabetes Mellitus during illness in children and young people under 18 years (Sick Day Rules) 2015 (21)

Figure 1 Management of hyperglycaemia. BG, blood glucose; CSII, continuous subcutaneous insulin infusion; DKA, diabetic ketoacidosis. 


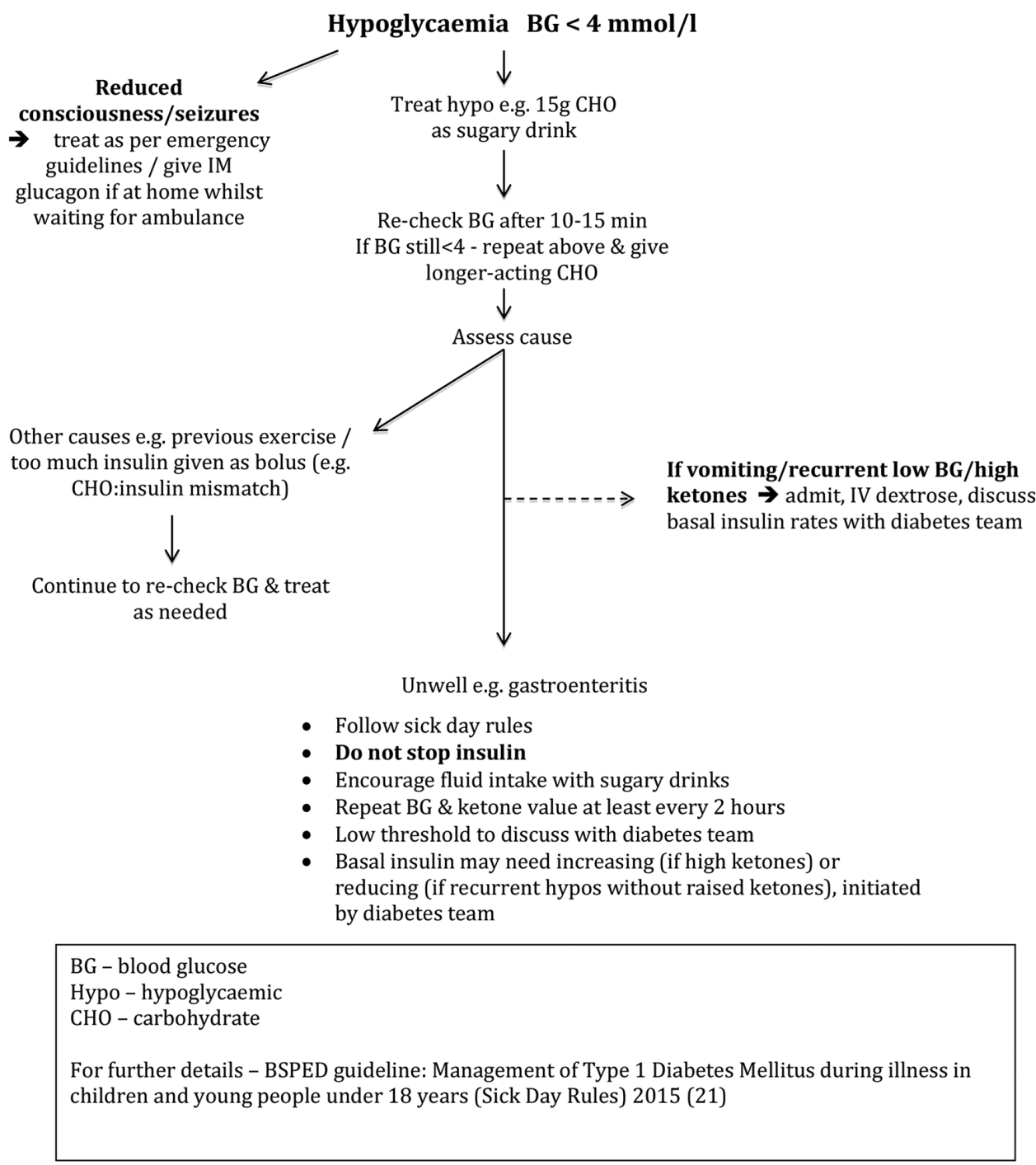

Figure 2 Management of hypoglycaemia.

\section{Box 3 Rules/practical tips}

\section{How to calculate correction dose/insulin sensitivity factor (ISF): \\ - '100' rule - 100/pump TDD \\ - For example, if the TDD is 20 , the ISF is $100 / 20=5$. \\ - So, 1 unit of insulin will reduce blood glucose by $5 \mathrm{mmol} / \mathrm{L}$.}

\section{How to calculate approximate insulin:carbohydrate} ratio:

- '500' rule $-500 /$ pump TDD=insulin to carb ratio

- For example, if the TDD is 20 , the ratio is $500 / 20=25$.

- That is, 1 unit insulin should be given with $25 \mathrm{~g}$ carbohydrate.

- Or ' 300 ' rule is often used instead for young children.

To convert $\mathrm{HbA} 1 \mathrm{c} \mathrm{mmol} / \mathrm{mol}$ to \%:

http://www.diabetes.co.uk/hba1c-units-converter.html. improvement, ketone levels are rising or the child is vomiting, they will need admission.

Problems with CSII

In hyperglycaemia, checks should always be made for battery failure, disconnection or tubing occlusion. If air is seen in the tubing, it should be disconnected and the CSII 'prime' cycle used to prime the tubing with insulin, removing air bubbles. All manufacturing companies have a telephone support service for families (normally 24 hours) and can troubleshoot other problems.

\section{Hypoglycaemia-BG value $<4 \mathrm{mmol} / \mathrm{L}$}

Hypoglycaemia must be treated immediately with fastacting carbohydrate, or with intravenous dextrose if severe. All families are provided with an emergency kit containing glucose, and intramuscular glucagon to use if the child is not able to take oral glucose, while 
waiting for an ambulance. Hypoglycaemia is most commonly a result of intercurrent illness, particularly gastroenteritis, or insulin:carbohydrate mismatch (figure 2).

It is often difficult to manage gastroenteritis in a child/young person with T1D, especially if their oral intake is reduced. It is important not to stop the insulin, and again families will follow the 'sick day rules'. (Sometimes changes are made to the temporary basal rate, by the diabetic team.) However, additional glucose intake is needed, and sips of sugar-containing fluids should be given frequently. Blood ketone levels must be checked regularly and 'starvation' ketones may be raised with vomiting and reduced oral intake, needing increased fluid in addition to glucose. If this is not possible or the child is vomiting, admission and intravenous dextrose will be needed.

\section{CONCLUSION}

CSII use has changed diabetes management, improving glucose control and quality of life for children/young people and their families. However, it brings added complexity to acute paediatric care, requiring specialist input. Sick day rules and national guidelines should be followed, and insulin should not be stopped, even in intercurrent illness.

Contributors PP wrote the first draft. DE contributed to the writing and
review of the paper. Competing interests None declared.

Provenance and peer review Commissioned; externally peer reviewed.

(C) Article author(s) (or their employer(s) unless otherwise stated in the text of the article) 2018. All rights reserved. No commercial use is permitted unless otherwise expressly granted.

\section{REFERENCES}

1 Cameron FJ, Wherrett DK. Care of diabetes in children and adolescents: controversies, changes, and consensus. Lancet 2015;385:2096-106.

2 Nathan DM, Genuth S, Lachin J, et al. The effect of intensive treatment of diabetes on the development and progression of long-term complications in insulin-dependent diabetes mellitus. N Engl J Med 1993;329:977-86.

3 White NH, Cleary PA, Dahms W, et al. Beneficial effects of intensive therapy of diabetes during adolescence: outcomes after the conclusion of the Diabetes Control and Complications Trial (DCCT). J Pediatr 2001;139:804-12.

4 Tamborlane WV, Sherwin RS, Genel M, et al. Reduction to normal of plasma glucose in juvenile diabetes by subcutaneous administration of insulin with a portable infusion pump. $N$ Engl J Med 1979;300:573-8.

5 Sherr JL, Hermann JM, Campbell F, et al. Use of insulin pump therapy in children and adolescents with type 1 diabetes and its impact on metabolic control: comparison of results from three large, transatlantic paediatric registries. Diabetologia 2016;59:87-91.
6 Ghatak A, Paul P, Hawcutt DB, et al. UK service level audit of insulin pump therapy in paediatrics. Diabet Med 2015;32:1652-7.

7 Miller KM, Foster NC, Beck RW, et al. Current state of type 1 diabetes treatment in the U.S.: updated data from the T1D Exchange clinic registry. Diabetes Care 2015;38:971-8.

8 Hussain S, Oliver N. Insulin pumps and continuous glucose monitoring made easy. The Netherland: Elsevier, 2016.

9 Abdullah N, Pesterfield C, Elleri D, et al. Management of insulin pump therapy in children with type 1 diabetes. Arch Dis Child Educ Pract Ed 2014;99:214-20.

10 Phillip M, Battelino T, Rodriguez H, et al. Use of insulin pump therapy in the pediatric age-group: consensus statement from the European Society for Paediatric Endocrinology, the Lawson Wilkins Pediatric Endocrine Society, and the International Society for Pediatric and Adolescent Diabetes, endorsed by the American Diabetes Association and the European Association for the Study of Diabetes. Diabetes Care 2007;30:1653-62.

11 Golden SH, Brown T, Yeh HC, et al. Methods for Insulin Delivery and Glucose Monitoring: Comparative Effectiveness. Rockville: Agency for Healthcare Research and Quality, 2012.

12 Pozzilli P, Battelino T, Danne T, et al. Continuous subcutaneous insulin infusion in diabetes: patient populations, safety, efficacy, and pharmacoeconomics. Diabetes Metab Res Rev 2016;32:21-39.

13 Pickup JC, Sutton AJ. Severe hypoglycaemia and glycaemic control in Type 1 diabetes: meta-analysis of multiple daily insulin injections compared with continuous subcutaneous insulin infusion. Diabet Med 2008;25:765-74.

14 Szypowska A, Schwandt A, Svensson J, et al. Insulin pump therapy in children with type 1 diabetes: analysis of data from the SWEET registry. Pediatr Diabetes 2016;17(suppl 23):3845.

15 National Institute for Health and Care Excellence. Continuous subcutaneous insulin infusion for the treatment of diabetes mellitus. Technology appraisal guidance [TA151]. 2008 https:// www.nice.org.uk/guidance/ta151.

16 International Diabetes Federation. Global IDF/ISPAD Guideline for Diabetes in Childhood and Adolescence. Germany: ISPAD Executive Office, 2011. http://c.ymcdn.com/sites/ispad.site-ym. com/resource/resmgr/Docs/idf-ispad_guidelines_2011_0.pdf.

17 Thabit H, Hovorka R. Coming of age: the artificial pancreas for type 1 diabetes. Diabetologia 2016;59:1795-805.

18 Ross PL, Milburn J, Reith DM, et al. Clinical review: insulin pump-associated adverse events in adults and children. Acta Diabetol 2015;52:1017-24.

19 Edge JA. BSPED Recommended Guideline for the Management of Children and Young People under the age of 18 years with Diabetic Ketoacidosis, 2015. http://www.bsped. org.uk/clinical/doCS/DKAguideline.pdf.

20 National Institute for Health and Care Excellence. Diabetes (type 1 and type 2 ) in children and young people: diagnosis and management (NG18), 2015. https://www.nice.org.uk/ guidance/ng18.

$21 \mathrm{Ng} \mathrm{SM}$, Soni A, Agwu JC, et al. Management of Type 1 Diabetes Mellitus during illness in children and young people under 18 years (Sick Day Rules), 2015. http://www.bsped.org. uk/clinical/docs/Sick_day_guideline_Main.pdf. 